\title{
Dependence of radio halos on underlying star formation activity and galaxy mass
}

\author{
U. Lisenfeld ${ }^{1,2}$, M. Dahlem ${ }^{3}$ and J. Rossa ${ }^{4}$ \\ ${ }^{1}$ Dept. Física Teórica y del Cosmos, Universidad de Granada, Spain \\ email: ute@ugr.es \\ ${ }^{2}$ Instituto de Astrofísica de Andalucía (IAA/CSIC), Apdo. 3004, 18080 Granada, Spain \\ ${ }^{3}$ CSIRO/ATNF, Paul Wild Observatory, Locked Bag 194, Narrabri NSW 2390, Australia \\ ${ }^{4}$ Department of Astronomy, University of Florida, 211 Bryant Space Science Center, P.O. Box \\ 112055, Gainesville, FL 32611-2055, U.S.A.
}

\begin{abstract}
We investigate the relation between the existence and size of radio halos, which are believed to be created by star formation (SF) related energy input into the interstellar medium, and other galaxy properties, most importantly star formation activity and galaxy mass. Based on radio continuum and $\mathrm{H} \alpha$ observations of a sample of seven galaxies we find a direct, linear correlation of the radial extent of gaseous halos on the size of the actively star-forming parts of the galaxy disks. Data of a larger sample of 22 galaxies indicate that the threshold energy input rate into the disk ISM per unit surface area for the creation of a gaseous halo depends on the mass surface density of the galaxy, in the sense that a higher (lower) threshold has to be surpassed for galaxies with a higher (lower) surface density. Because of the good prediction of the existence of a radio halo from these two parameters, we conclude that they are important, albeit not the only contributors. The compactness of the SF-related energy input is also found to be a relevant factor. Galaxies with relatively compact SF distributions are more likely to have gaseous halos than others with more widespread SF activity. These results quantify the so-called "break-out" condition for matter to escape from galaxy disks, as used in all current models of the interstellar medium.
\end{abstract}

More details can be found Dahlem, Lisenfeld \& Rossa, 2006, A\&A 457, 121.

Keywords. ISM: general - galaxies: spirals - galaxies: evolution - galaxies: halos - galaxies: starburst - radio continuum: galaxies 